\title{
Primary High-grade Serous Carcinoma of the Fallopian Tube as an Incidental Intraoperative Finding: A Case Report
}

\author{
Nasim Shokouhi ${ }^{1}$, Sara Saeedi ${ }^{1}$, Soheila Sarmadi ${ }^{2}$, Behnaz Moradi $\left(\mathbb{D}^{3}\right.$ and Elham Feizabad ${ }^{1,{ }^{*}}$ \\ ${ }^{1}$ Department of Obstetrics and Gynecology, Yas Hospital, Tehran University of Medical Sciences, Tehran, Iran \\ ${ }^{2}$ Department of Pathology, Yas Hospital, Tehran University of Medical Sciences, Tehran, Iran \\ ${ }^{3}$ Department of Radiology, Yas Hospital, Tehran University of Medical Sciences, Tehran, Iran \\ Corresponding author: Department of Obstetrics and Gynecology, Yas Hospital, Tehran University of Medical Sciences, Tehran, Iran. Email: elhamfeizabad@gmail.com
}

Received 2021 February 08; Revised 2021 September 05; Accepted 2021 September 06.

\begin{abstract}
Primary carcinoma of the fallopian tube is a rare, but fatal gynecologic cancer. The preoperative diagnosis of this carcinoma is challenging due to the absence of specific symptoms and signs, and in most patients, it is an intraoperative finding. A 55-year-old patient (G3Ab1P2) was referred to the urogynecology clinic of our hospital with the chief complaints of heavy, prolonged menstrual bleeding and a persistently abnormal yellow discharge, which could not be distinguished by the patient from urinary leaks. After a complete diagnostic work-up, the patient was identified as a candidate for hysterectomy due to abnormal vaginal bleeding resistant to megestrol acetate, family history of malignancy, and abnormal vaginal discharge. Laparotomy revealed unusual left fallopian tube features (large, bulky, and vegetative), suggesting malignancy. The intraoperative frozen-section analysis of the left fallopian tube and the ovarian specimens indicated the mass as a high-grade serous carcinoma of the fallopian tube. Total abdominal hysterectomy, bilateral salpingo-oophorectomy, and partial omentectomy were performed for the patient. The definitive histopathological diagnosis was high-grade serous carcinoma of the left fallopian tube (stage $2 \mathrm{~b}$ ) with omental involvement, without any evidence of lymphovascular invasion. High-grade serous carcinoma of the fallopian tube is likely to have non-specific symptoms, causing a significant delay in diagnosis and treatment, which negatively affects the prognosis and survival of these patients.
\end{abstract}

Keywords: Magnetic Resonance Imaging, Serous, Transvaginal Ultrasound, Fallopian Tubes, Cystadenocarcinoma

\section{Introduction}

Primary carcinoma of the fallopian tube is a very unusual, yet lethal gynecologic malignancy, which accounts for less than $1 \%$ of all malignancies of the female reproductive system (1). The incidence of this cancer dramatically increases in middle-aged women, reaching its peak in the age range of 60 - 64 years (2). Preoperative diagnosis is usually challenging due to the absence of specific symptoms and signs, and in most cases, it is an intraoperative finding or a histopathological diagnosis (3).

Herein, we present a case of primary high-grade serous carcinoma of the fallopian tube with different clinical presentations. Diagnosis was made incidentally during a total hysterectomy because of drug-resistant endometrial hyperplasia, persistent spotting, and abnormal vaginal discharge.

\section{Case Presentation}

A 55-year-old woman (G3Ab1P2) with a history of treated hypertension and hypothyroidism presented to our urogynecology clinic with chief complaints of urinary incontinence, in addition to two years of heavy menstrual bleeding. She reported sexual activity and a positive family history of uterine malignancy in her daughter and her sister, who underwent total abdominal hysterectomy (TAH). The patient's medical history revealed mixed urinary incontinence with urge-predominant symptoms. Besides, the physical examination revealed stage I pelvic organ prolapse in all three compartments and a positive cough stress test. The results of transvaginal ultrasound (TVS) and endometrial biopsy were normal.

Moreover, a Papanicolaou smear test was carried out, the result of which was negative for intraepithelial lesions or malignancies. To relieve the urinary symptoms, an anticholinergic medication was prescribed, and the patient was asked to return for follow-ups in one month to evaluate her menstrual bleeding pattern after endometrial biopsy and track her response to the anticholinergic medication. However, the patient did not return for the follow-up visit. One year later, she presented to our hospital with the chief complaints of heavy, prolonged menstrual bleeding and a persistently abnormal yellow dis- 
charge that could not be distinguished by the patient from urinary leaks. On examination, no pelvic mass was palpable. Accordingly, mixed vaginal infection and uterine prolapse stage I were diagnosed.

The patient underwent TVS, which indicated a $21 \times 18$ $\mathrm{mm}$ endometrial cyst in the right ovary and a $32 \times 18 \mathrm{~mm}$ tabular hypoechoic structure with a hypervascular thick wall in the left adnexa (separated from the left ovary), suggesting a left fallopian tube pathology. For further assessments, magnetic resonance imaging (MRI) was requested. The MRI-enhanced images of the pelvic cavity confirmed a small endometrial cyst in the right ovary, as well as a tubular left adnexal structure, measuring $36 \times 12 \mathrm{~mm}$, with thick rim enhancement, without internal solid components, strongly suggesting hematosalpinx, hydrosalpinx, or an inflammatory process, without any evidence of malignancy (Figure 1).

Focal hyperplasia without atypia with a disordered proliferative endometrium was detected on hysteroscopy (D \& C). The abnormal laboratory results included a hemoglobin level of $10.9 \mathrm{~g} / \mathrm{dL}$ and an erythrocyte sedimentation rate (ESR) of 97. Finally, the patient was introduced as a candidate for TAH and bilateral salpingooophorectomy (BSO) due to abnormal vaginal bleeding resistant to megestrol acetate, a family history of malignancy, and abnormal vaginal discharge. Before surgery, an urodynamic study was also carried out because of urgencypredominant mixed urinary incontinence and the patient's inability to differentiate vaginal discharge from urinary leaks. The urodynamic study revealed normal findings.

Laparotomy revealed unusual left fallopian tube features (large, bulky, and vegetative), suggesting malignancy. The intraoperative frozen-section analysis of the left fallopian tube and the ovarian specimens detected the mass as a high-grade serous carcinoma of the fallopian tube. Total abdominal hysterectomy, BSO, and partial omentectomy were performed for the patient. The definitive histopathological diagnosis was high-grade serous carcinoma of the left fallopian tube (stage $2 \mathrm{~b}$ ) (Figure 2) with omental involvement, without any evidence of lymphovascular invasion. Considering the normal postoperative MRI findings, six courses of adjuvant chemotherapy with carboplatin and paclitaxel, followed by active surveillance, were prescribed for the patient. During this report (one month after surgery), she was receiving one course of chemotherapy, without any major adverse events.

\section{Discussion}

This report presented an uncommon case of highgrade serous carcinoma of the fallopian tube (stage $2 b$ ). Our patient ( 55 years old) initially complained of abnormal vaginal bleeding and abnormal vaginal discharge; however, she could not distinguish the vaginal discharge from urinary leaks.

Although little is known about the etiology of the fallopian tube carcinoma, it seems that hormonal, reproductive, and genetic factors, besides the presence of pelvic chronic inflammation, are possible causes (4). Our patient did not have a history of oral contraceptive pill (OCP) use, infertility, and Tubal ligation (TL), although she had a recent history of recurrent, drug-resistant vaginal infections, along with chronic salpingitis in the pathology report. According to the literature review in the present study, abnormal vaginal bleeding and vaginal discharge were the most common symptoms of the fallopian tube carcinoma. Although the physical examination of our patient did not reveal a palpable pelvic mass, it may be detected in some patients (5).

Generally, primary carcinoma of the fallopian tube is difficult to diagnose before surgery due to the absence of specific symptoms and signs. In a previous study, although abnormal vaginal bleeding or discharge, palpable pelvic mass, and abdominal pain comprised a diagnostic triad for carcinoma of the fallopian tube, this triad could be only observed in 5 to $20 \%$ of cases (6). It is worth mentioning that our patient was unable to differentiate urinary incontinence from vaginal discharge, which led to her delayed diagnosis.

Some studies have recently suggested that TVS and serum cancer antigen 125 (CA-125) assay can be useful for the preoperative detection of this malignancy $(7,8)$. However, imaging without identifying the specific features of the fallopian tube carcinoma cannot be a perfect guide for preoperative diagnosis. Similarly, in our patient, TVS and even MRI did not lead to a preoperative diagnosis. The CA125 antigen is mostly expressed by the fallopian tube carcinoma, and high serum levels of this antigen have been found in patients with advanced or recurrent malignancy (9). During surgery, the increased pelvic spread of the fallopian tube carcinoma has been observed in 18 to $60 \%$ of the patients. Besides, bilateral tubal involvement has been detected in $10-27 \%$ of the cases (10).

The overall five-year survival of patients with primary carcinoma of the fallopian tube ranges from 14 to $57 \%$. The disease extent, stage of disease, residual disease, and histological grade after the initial surgery are important prognostic factors in determining the patient's prognosis (10). However, further research is required for a timely diagnosis. The present findings emphasized that immediate detection contributes to the measurement of inflammatory markers in the vagina from the fallopian tube, showing probable chronic inflammation, which is a risk factor for serous carcinoma. Besides, the serum CA-125 level is a useful non-invasive marker for controlling the fallopian tube carcinoma (11). 

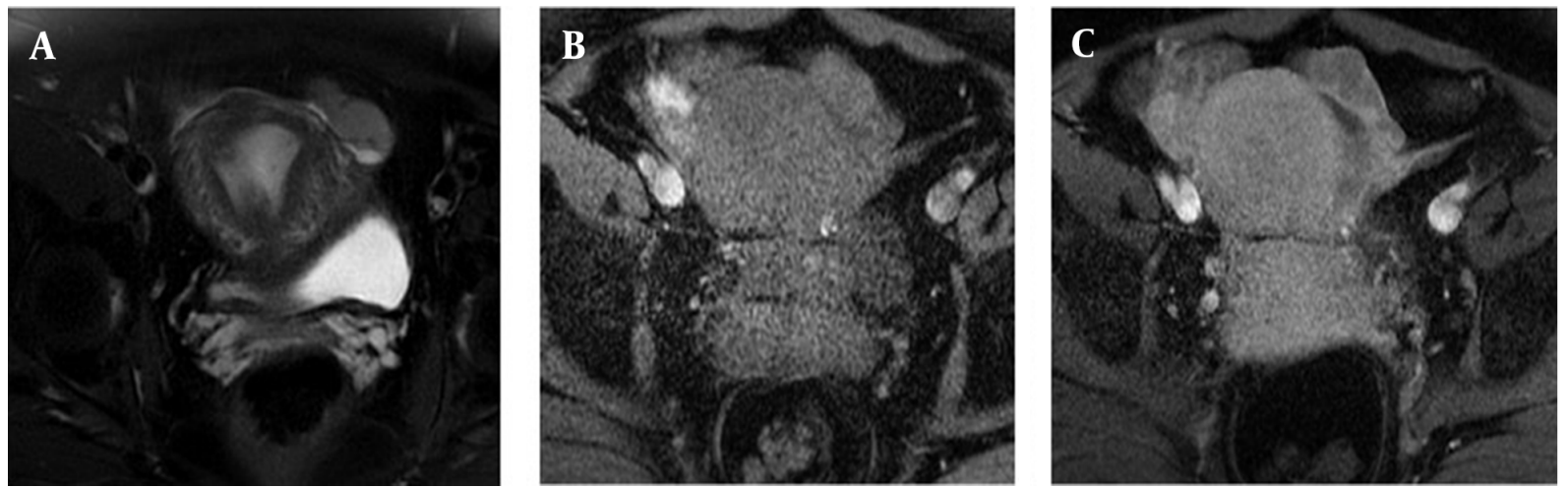

Figure 1. The MRI findings of a 55-year-old patient with the chief complaints of heavy, prolonged menstrual bleeding and a persistently abnormal yellow discharge. The definitive histopathological diagnosis was high-grade serous carcinoma of the left fallopian tube. A, T2-weighted image shows a tubular, slightly low-signal structure in the left adnexa, separated from the left ovary. B, This tubular structure shows iso-signals in the T1-weighted image with only a thick wall enhancement pattern in the post-contrast image. C, There is no obvious solid component enhancement.
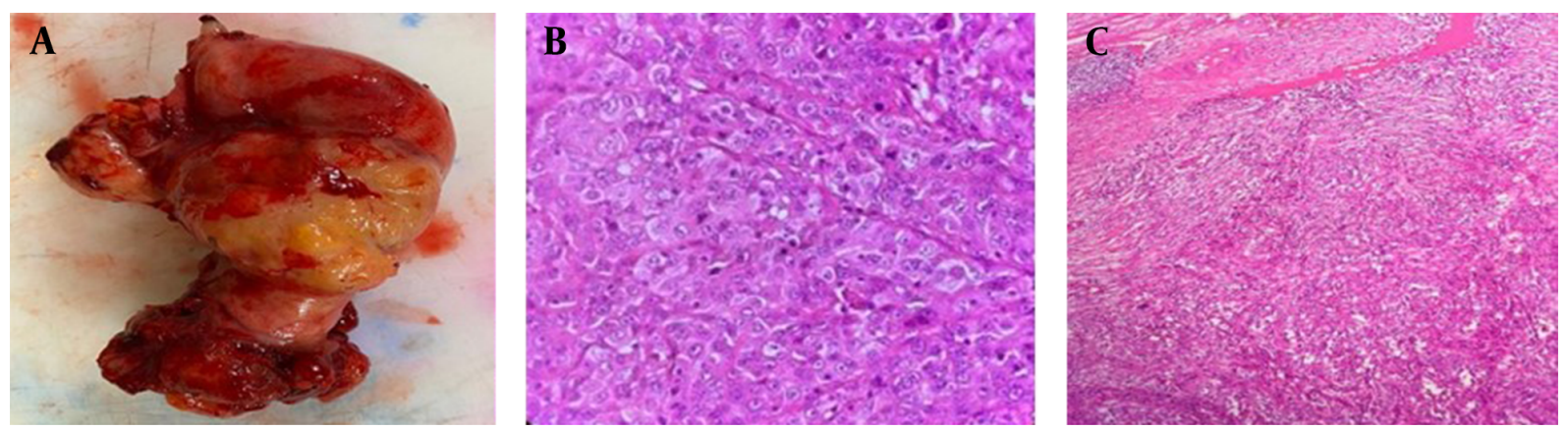

Figure 2. The histological structure of the tumor. A, The macroscopic view of the left fallopian tube. B, High-grade serous carcinoma with solid growth, marked cytologic atypia, and high mitotic activity. C,The tumor infiltrated into the fallopian tube wall in the presence of chronic salpingitis.

The present findings demonstrated that high-grade serous carcinoma of the fallopian tube has non-specific symptoms, which may lead to a significantly delayed clinical diagnosis and treatment and adversely affect the prognosis and survival of the patients.

\section{Footnotes}

Authors' Contribution: All authors contributed to the manuscript. N.Sh. and S.S. (Sara Saeedi) performed the surgery; S.S. (Soheila Sarmadi) performed the histological examinations; B.M. contributed to the diagnostic decisionmaking and writing the manuscript. E.F. and N.Sh. were mainly responsible for writing the manuscript. All authors read and approved the final manuscript.

Conflict of Interests: The authors declare no conflicts of interest.

Funding/Support: The authors received no financial support for this study.
Informed Consent: The patient completed an informed consent form for the anonymous publication of the data.

\section{References}

1. Tounsi N, Bouazize H, Margheli I, Boujelbene N, Monia H, Slimane $\mathrm{M}$, et al. Primary fallopian tube carcinoma: Results of a singleinstitutional retrospective analysis of 17 patients with evaluation of staging and prognostic factors. Obstet Gynaecol Reprod Med. 2021;31:14. doi: 10.21613 /gorm.2021.1075.

2. Al-Agha OM, Blake Gilks C. High-grade serous carcinoma involving fallopian tube, ovary and peritoneum. Surg Pathol Clin. 2011;4(1):375-96. doi: 10.1016/j.path.2010.12.004. [PubMed: 26837299].

3. Morrison JC, Blanco LJ, Vang R, Ronnett BM. Incidental serous tubal intraepithelial carcinoma and early invasive serous carcinoma in the nonprophylactic setting: Analysis of a case series. Am J Surg Pathol. 2015;39(4):442-53. doi: 10.1097/PAS.0000000000000352. [PubMed: 25517955].

4. Veloso Gomes F, Dias JL, Lucas R, Cunha TM. Primary fallopian tube carcinoma: Review of MR imaging findings. Insights Imaging. 2015;6(4):431-9. doi: 10.1007/s13244-015-0416-y. [PubMed: 26150249]. [PubMed Central: PMC4519813].

5. Dhakhwa R, Vaidya A, Giri A, Shakya A, Vaidya A. Primary high grade serous carcinoma of the fallopian tube: A case report. JNMA J Nepal 
Med Assoc. 2020;58(231):927-9. doi: 10.31729/jnma.5674. [PubMed: 34506403]. [PubMed Central: PMC7775018].

6. Lacy MQ, Hartmann LC, Keeney GL, Cha SC, Wieand HS, Podratz KC, et al. c-erbB-2 and p53 expression in fallopian tube carcinoma. Cancer. 1995;75(12):2891-6. doi: 10.1002/1097-0142(19950615)75:12<2891::aidcncr2820751216>3.0.co;2-b. [PubMed: 7773939].

7. Ekici E, Vicdan K, Danisman N, Soysal ME, Cobanoglu O, Gokmen O. Ultrasonographic appearance of fallopian tube carcinoma. Int J Gynaecol Obstet. 1995;49(3):325-9. doi: 10.1016/0020-7292(95)02342-a. [PubMed: 9764874].

8. KurjakA, Kupesic S, Ilijas M, Sparac V, Kosuta D. Preoperative diagnosis of primary fallopian tube carcinoma. Gynecol Oncol. 1998;68(1):29-34. doi: 10.1006/gyno.1997.4873. [PubMed: 9454656].
9. Mi D, Zhang Y. Prognostic value of serum HE4 in patients with advanced ovarian, fallopian tube, and peritoneal carcinoma. Arch Gynecol Obstet. 2020;301(3):779-85. doi: 10.1007/s00404-020-05447-z. [PubMed: 32034508].

10. Gadducci A, Landoni F, Sartori E, Maggino T, Zola P, Gabriele A, et al Analysis of treatment failures and survival of patients with fallopian tube carcinoma: A cooperation task force (CTF) study. Gynecol Oncol. 2001;81(2):150-9. doi:10.1006/gyno.2001.6134. [PubMed: 11330942].

11. Salvador S, Gilks B, Kobel M, Huntsman D, Rosen B, Miller D The fallopian tube: primary site of most pelvic high-grade serous carcinomas. Int J Gynecol Cancer. 2009;19(1):58-64. doi: 10.1111/IGC.0b013e318199009c. [PubMed: 19258943]. 